\title{
A Generalization Concept of Measure by Using Binary Operations
}

\author{
M-Hassan Hadadzadeh Naiery ${ }^{1}$ \\ ${ }^{1}$ Department of Mathematics, Faculty of Sciences, Semnan University, Semnan, Iran \\ Correspondence: M-Hassan Hadadzadeh Naiery, Department of Mathematics, Faculty of Sciences, Semnan \\ University, Semnan, Iran.
}

Received: May 27, 2017

doi:10.5539/mas.v11n11p85
Accepted: June 8, 2017 Online Published: October 31, 2017

URL: https://doi.org/10.5539/mas.v11n11p85

\section{Abstract}

In this paper, a generalization of the concept of measure is made using binary operations.

With this generalization, we extend the measure domain to $[0, \infty) \times 2^{X}$. For this purpose, we define function $\mu:[0, \infty) \times 2^{X} \rightarrow \mathbb{R}^{0}$, which simultaneously satisfies the following two conditions:

$$
\begin{gathered}
\mu\left(x, \cup_{i=0}^{\infty} A_{i}\right)=\sum_{i=0}^{\infty} \mu\left(x, A_{i}\right) \quad A_{i} \cap A_{j}=\emptyset \quad i \neq j \\
\mu(x, A) \otimes \mu(y, B) \leq \mu(x \boxplus y, A \cup B)
\end{gathered}
$$

Such that in second condition $\otimes, \boxplus$ are binary operations.

In the future, the properties of this new concept will be examined.

In this paper, there are correlations between algebraic concepts (such as binary operation) and analysis (such as measure). Of course, in this article we pay more attention to algebraic concepts, in other words, the properties of binary operation created by this new definition are more highly evaluated.

Keywords: measure, dependable value pre-measure, dependable value measure, dependable value measure under binary oprations

\section{Introduction}

When we study of measure theory, we understand this formal theory is using in Lebesgue and Integral operation. In the following, the sigma algebra structure is proper to implement. As we know, when we talk about measure, we mean the function such as; $\vartheta: 2^{X} \rightarrow \mathbb{R}^{0}$ which has the following properties:

$$
\left\{\vartheta\left(\bigcup_{i=0}^{\infty} A_{i}\right)=\sum_{i=0}^{\infty} \vartheta\left(A_{i}\right) \quad A_{i} \cap A_{j}=\emptyset i \neq j\right.
$$

The meaning of $\mathbb{R}^{0}$ is the non -negative real numbers. The triple $(X, S, \vartheta)$ is which $X$ is an nonempty set and $S$ is ring of Subset of $X, \vartheta$ that's a measure of $S$ sigma algebra. It is called measure space. In this article, we have generalized the measure range from $2^{X}$ to $\mathbb{R}^{0} \times 2^{X}$. The main principle, which used in this purpose, are binary operations. For this purpose, we define function $\mu: \mathbb{R}^{0} \times 2^{X} \rightarrow \mathbb{R}^{0}$, which simultaneously satisfies the following two conditions:

$$
\begin{gathered}
\mu\left(x, \bigcup_{i=0}^{\infty} A_{i}\right)=\sum_{i=0}^{\infty} \mu\left(x, A_{i}\right) \quad A_{i} \cap A_{j}=\emptyset \quad i \neq j \\
\mu(x, A) \otimes \mu(y, B) \leq \mu(x \boxplus y, A \bigcup B)
\end{gathered}
$$

Such that in second condition $\otimes, \boxplus$ are binary operations. The easiest way to satisfy the function in the first condition is $\mu: \mathbb{R}^{0} \times 2^{X} \rightarrow \mathbb{R}^{0}$, defines as follow :

$$
\mu(x, A)=f(x) \vartheta(A)
$$

Such that $\vartheta: S \rightarrow \mathbb{R}^{0}$ is a measure and $f: \mathbb{R}^{0} \rightarrow \mathbb{R}^{0}$ is a function. The function that satisfies the first condition 
is called the dependable value pre-measure. In addition, when binary operations defined as the sum, this measure is called dependable value measure.

This dependable value pre-measure which satisfy in condition 2 is named dependable value measure under binary operations $(\otimes, \boxplus)$.In the following, we will examine the conditions which dependable value pre-measure is turned to the dependable value measure. Finally, we recognize some properties of this type of measures. It seems that we can define a numerous advantages for the generalization of the measure theory of $2^{X}$ to $\mathbb{R}^{0} \times 2^{X}$. One uses of this type of measure are in a nonlinear analysis.

Definition $1 . \mu: \mathbb{R}^{0} \times 2^{X} \rightarrow \mathbb{R}^{0}$ is dependable value pre-measure, If $\mu$ is a function that we define as follows:

$$
\mu\left(x, \bigcup_{i=0}^{\infty} A_{i}\right)=\sum_{i=0}^{\infty} \mu\left(x, A_{i}\right) \quad A_{i} \cap A_{j}=\emptyset \quad i \neq j
$$

The easiest way to make a pre-measure is that function $\mu: \mathbb{R}^{0} \times 2^{X} \rightarrow \mathbb{R}^{0}$ define as follows:

$$
\mu(x, A)=f(x) \vartheta(A)
$$

Such that $\vartheta: 2^{X} \rightarrow \mathbb{R}^{0}$ is measure and $f: \mathbb{R}^{0} \rightarrow \mathbb{R}^{0}$ is function. In all of this paper, it's assumed that the function $\mu: \mathbb{R}^{0} \times 2^{X} \rightarrow \mathbb{R}^{0}$ is make in this way.

dependable value pre-measure $\mu: \mathbb{R}^{0} \times 2^{X} \rightarrow \mathbb{R}^{0}$ that is called a dependable value measure under binary operations $(\otimes, \boxplus)$, If $\mu$ is function as follows:

Such that $\otimes, \boxplus: \mathbb{R}^{0} \times \mathbb{R}^{0} \rightarrow \mathbb{R}^{0}$ are binary oprations.

$$
\mu(x, A) \otimes \mu(y, B) \leq \mu(x \boxplus y, A \bigcup B)
$$

If binary operations $\boxplus$ and $\otimes$ are simple sum, this function is called dependable value measure. In other words, each dependable value measure is a function, such as $\rho: \mathbb{R}^{0} \times 2^{X} \rightarrow \mathbb{R}^{0}$ which satisfies to the following properties:

$$
\begin{gathered}
\rho\left(x, \bigcup_{i=0}^{\infty} A_{i}\right)=\sum_{i=0}^{\infty} \rho\left(x, A_{i}\right) \quad A_{i} \cap A_{j}=\varnothing \quad i \neq j \\
\rho(x, A)+\rho(y, B) \leq \rho(x+y, A \bigcup B)
\end{gathered}
$$

Example 1: let's assume that binary relations $\boxplus, \otimes: \mathbb{R}^{0} \times \mathbb{R}^{0} \rightarrow \mathbb{R}^{0}$, define as follows:

$$
\begin{aligned}
& a \otimes b=\min \{a, b\} \\
& a \boxplus b=\max \{a, b\}
\end{aligned}
$$

In addition, $\vartheta$ on measure space can define as $(X, S, \vartheta)$. In this case, the function $\mu_{1}: \mathbb{R}^{0} \times 2^{X} \rightarrow \mathbb{R}^{0}$ which is define as follows and it's a measure under binary operations $(\otimes, \boxplus)$.

$$
\mu_{1}(x, A)=x . \vartheta(A)
$$

Example 2: let's assume that binary relations $\boxplus, \otimes: \mathbb{R}^{0} \times \mathbb{R}^{0} \rightarrow \mathbb{R}^{0}$, define as follows:

$$
\begin{gathered}
a \otimes b=5 a+7 b \\
a \boxplus b=6 a+7 b+a^{2 b}
\end{gathered}
$$

In addition, $\vartheta$ on measure space can define as $(X, S, \vartheta)$. In this case, the function is $\mu_{2}: \mathbb{R}^{0} \times 2^{X} \rightarrow \mathbb{R}^{0}$ which is define as follows and it's a measure under binary operations $(\otimes, \boxplus)$.

$$
\mu_{2}(x, A)=x \cdot \vartheta(A)
$$

Example 3: The function $\rho_{1}: \mathbb{R}^{0} \times 2^{X} \rightarrow \mathbb{R}^{0}$ is a dependable value measure.

$$
\rho_{1}(x, A)=\left\{\begin{array}{lc}
0 & x=0 \text { or } A=\emptyset \\
\infty & \text { Otherwise }
\end{array}\right.
$$

Theorem1.If $\vartheta$ is a measure on space $(X, S, \vartheta)$. In addition, if $\varphi: \mathbb{R}^{0} \rightarrow \mathbb{R}^{0}$ is a function it satisfies on this condition:

$$
\varphi(a) \otimes \varphi(b) \leq \varphi(a \boxplus b)
$$

And a binary operation $\otimes: \mathbb{R}^{0} \times \mathbb{R}^{0} \rightarrow \mathbb{R}^{0}$ should be like for any $a, b \in \mathbb{R}^{0}$, so we have as follow : 


$$
\max \{a, b\} \leq a \otimes b \leq a+b
$$

Moreover, a binary operation $\Delta: \mathbb{R}^{0} \times \mathbb{R}^{0} \rightarrow \mathbb{R}^{0}$ should be like for any $c, d, e, f, g \in \mathbb{R}^{0}$ with $f \geq g$ condition, so we have as follow:

$$
c \Delta(d+e)=(c \Delta d)+(c \Delta e)
$$

$$
f \Delta e \geq g \Delta e
$$

Also, if we define a function like this $\mu: \mathbb{R}^{0} \times 2^{X} \rightarrow \mathbb{R}^{0}$ as follow :

$$
\mu(x, A)=\varphi(x) \Delta \vartheta(A)
$$

Then, $\mu: \mathbb{R}^{0} \times 2^{X} \rightarrow \mathbb{R}^{0}$ is a dependable value measure under binary operations $(\otimes, \boxplus)$.

Proof. The proof that function $\mu: \mathbb{R}^{0} \times 2^{X} \rightarrow \mathbb{R}^{0}$ is dependable value pre-measure, it is easily obtained by using (2) and (4). In order to proof that function is dependable value measure under binary operations $(\otimes, \boxplus)$, it is easy to write down the properties of $\left(^{*}\right)$ and (1) and (3):

$$
\begin{gathered}
\mu(x, A) \otimes \mu(y, B) \leq \mu(x, A)+\mu(y, B) \\
=[\varphi(x) \Delta(\vartheta(A)]+[\varphi(y) \Delta \vartheta(B)] \\
\leq[\max \{\varphi(x), \varphi(y)\} \Delta(\vartheta(A)]+[\max \{\varphi(x), \varphi(y)\} \Delta(\vartheta(B)] \\
\leq[\varphi(x) \otimes \varphi(y)] \Delta(\vartheta(A))+[\varphi(x) \otimes \varphi(y)] \Delta(\vartheta(B)) \\
=[\varphi(x) \otimes \varphi(y)] \Delta(\vartheta(A)+\vartheta(B))=[\varphi(x) \otimes \varphi(y)] \Delta \vartheta(A \cup B) \\
\leq \varphi(x \boxplus y) \vartheta(A \cup B)=\mu(x \boxplus y, A \cup B)
\end{gathered}
$$

Corollary.If $\vartheta$ is a measure on space $(X, S, \vartheta)$, also $\varphi: \mathbb{R}^{0} \rightarrow \mathbb{R}^{0}$ is a function such that satisfies on the condition;

$$
\varphi(a)+\varphi(b) \leq \varphi(a+b)
$$

In addition, if we define a function like this $\boldsymbol{\rho}: \mathbb{R}^{0} \times 2^{X} \rightarrow \mathbb{R}^{0}$, as follow:

$$
\rho(x, A)=\varphi(x) \cdot \vartheta(A)
$$

Then $\rho: \mathbb{R}^{0} \times 2^{X} \rightarrow \mathbb{R}^{0}$ is dependable value measure.

In order to understand the theorem(1) and Corollary, we can give the following example.

Example 4: let's assume that $\vartheta: 2^{X} \rightarrow \mathbb{R}^{0}$ is a measure on $(X, S, \vartheta)$ space. In this case, $\rho_{3}: \mathbb{R}^{0} \times 2^{X} \rightarrow$ $\mathbb{R}^{0}, \rho_{4}: \mathbb{R}^{0} \times 2^{X} \rightarrow \mathbb{R}^{0}$ which define below, are dependable value measures.

$$
\begin{gathered}
\rho_{3}(x, A)=\frac{x^{4}}{x^{2}+5} \cdot \vartheta(A) \\
\rho_{4}(x, A)=\sum_{i=1}^{n} a_{i} x^{i} \cdot \vartheta(A) \quad a_{i} \geq 0, x \geq 0
\end{gathered}
$$

Solution. Suppose that $\varphi(x)=\frac{x^{4}}{x^{2}+5}$.

In order to measure of $\rho_{3}: \mathbb{R}^{0} \times 2^{X} \rightarrow \mathbb{R}^{0}$, we must notice that we can write like this :

$$
\begin{gathered}
\varphi(x+y)-\varphi(x)-\varphi(y)= \\
\frac{(x+y)^{4}}{(x+y)^{2}+5}-\frac{x^{4}}{x^{2}+5}-\frac{y^{4}}{y^{2}+5}= \\
\frac{2 x^{5} y^{3}+10 x^{5} y+4 x^{4} y^{4}+25 x^{4} y^{2}+2 x^{3} y^{5}+40 x^{3} y^{3}+100 x^{3} y+25 x^{2} y^{4}+150 x^{2} y^{2}+10 x y^{5}+100 x y^{3}}{x^{4} y^{4}+5 x^{4}+2 x^{3} y^{3}+10 x^{3} y+x^{2} y^{4}+15 x^{2} y^{2}+50 x^{2}+10 x y^{3}+50 x y+5 y^{4}+50 y^{2}+125}
\end{gathered}
$$

It is clear that non-negative of the above fraction, according to positive value of $x, y$. So, this function $\varphi(x)=\frac{x^{4}}{x^{2}+5}$ is satisfies on this condition (**). In order to $\rho_{4}: \mathbb{R}^{0} \times 2^{X} \rightarrow \mathbb{R}^{0}$ measure, the proof of $\varphi$ $(x)=\sum_{i=1}^{n} a_{i} x^{i}$ is satisfies on this condition $(* *)$.It is easy to solve, according to the inequality.

$$
\text { a. } x^{n}+a \cdot y^{n} \leq a \cdot(x+y)^{n} \quad n \in \mathbb{N} \& x, y \in \mathbb{R}^{0}
$$

Lemma 2.Let's assume that $\mu: \mathbb{R}^{0} \times 2^{X} \rightarrow \mathbb{R}^{0}$ be a dependable value measure under binary operations $(\otimes, \boxplus)$. 
In addition, suppose for binary operations $\boxplus, \otimes: \mathbb{R}^{0} \times \mathbb{R}^{0} \rightarrow \mathbb{R}^{0}$;

we have:

$$
\begin{gathered}
a \otimes b \geq a \\
a \boxplus b=a+b
\end{gathered}
$$

Moreover if $x, y$ are non-negative numbers which have $y \leq x$ relation;show that

$$
\mu(y, A) \leq \mu(x, A)
$$

Proof.Due to the feature of II, which means dependable value measure under binary operations $(\Theta, \boxplus)$, we can write as follows :

Now, we have as follow:

$$
\mu(y, A) \otimes \mu(x-y, A) \leq \mu(y \boxplus(x-y), A)=\mu(x, A)
$$

Therefore, we get desired results.

$$
\mu(y, A) \otimes \mu(x-y, A) \geq \mu(y, A)
$$

Corollary.Let's think there is $y \leq x$ relation between two non-negative numbers $x, y$. In this case, the following relationship is established in each dependable value measure $\rho: \mathbb{R}^{0} \times 2^{X} \rightarrow \mathbb{R}^{0}$.

$$
\rho(y, A) \leq \rho(x, A)
$$

Example 5: Let's suppose that $\vartheta, \theta: \mathbb{R}^{0} \times 2^{X} \rightarrow \mathbb{R}^{0}$ are dependable value measures. In this case, $\rho: \mathbb{R}^{0} \times 2^{X} \rightarrow$ $\mathbb{R}^{0}$ is dependable value measure which defined by the following criteria.

$$
\rho(x, A)=x^{2} \vartheta(x, A)+x^{3} \theta(x, A)
$$

The research of first feature is very easy. In order to consider of the feature of $I I$ for $x^{2} \vartheta(x, A)$, we have to write as follow:

$$
\begin{gathered}
x^{2} \vartheta(x, A)+y^{2} \vartheta(y, B) \leq\left(x^{2}+y^{2}\right)(\vartheta(x, A)+\vartheta(y, B)) \leq \\
\left(x^{2}+y^{2}\right)(\vartheta(x+y, A \cup B)) \leq \\
(x+y)^{2}(\vartheta(x+y, A \cup B))
\end{gathered}
$$

Due to the same operation in this phrase $x^{3} \theta(x, A)$, we can conclude as follow:

$$
\mu(x, A)+\mu(y, B) \leq \mu(x+y, A \cup B)
$$

In two above operations $(\otimes, \boxplus)$ are consider as an addition. However, if they wasn't an addition, it was so hard to solve. In below theorem, we are looking for functions, which is multiplied by their dependable value measure, the multiplication again become a dependable value measure function under binary operations.

Theorem 3.Let's assume that $\theta: \mathbb{R}^{0} \times 2^{X} \rightarrow \mathbb{R}^{0}$ is dependable value measure under binary operations $(\otimes, \boxplus)$. In addition, we have for non-negative function $f: \mathbb{R}^{0} \rightarrow \mathbb{R}^{0}$ and binary operations $(\otimes, \boxplus)$ :

$$
\begin{array}{r}
f(x) \otimes f(y) \leq f(x \boxplus y) \\
(a b) \otimes(c d) \leq(a \otimes c)(b \otimes d)
\end{array}
$$

then function $\mu: \mathbb{R}^{0} \times 2^{X} \rightarrow \mathbb{R}^{0}$ is dependable value measure under binary operations $(\otimes, \boxplus)$.

$$
\mu(x, A)=f(x) \theta(x, A)
$$

Proof.The consideration of dependable value pre-measure function $\mu: \mathbb{R}^{0} \times 2^{X} \rightarrow \mathbb{R}^{0}$ is so simple. Now, by using the features (5) and (6), we can write:

$$
\begin{gathered}
\mu(x, A) \otimes \mu(y, B)=f(x) \theta(x, A) \otimes f(y) \theta(y, B) \leq \\
{[f(x) \otimes f(y)][\theta(x, A) \otimes \theta(y, B)] \leq[f(x \boxplus y)][\theta(x \boxplus y, A \cup B)]=\mu(x \boxplus y, A \cup B)}
\end{gathered}
$$

As we can see in the above theorem, we can apply the conditions, which are possible to maintain the binary operations. Certainly, the condition isn't always like that. However, in order to change the condition, the binary operations are also changed. The following theorem has this kind of conditions.

Theorem 4.Let's $\theta, \vartheta: \mathbb{R}^{0} \times 2^{X} \rightarrow \mathbb{R}^{0}$ are dependable value measures under binary operations respectively $(\otimes, \boxplus),(\widehat{\otimes}, \widehat{\boxplus}) ;$ such that both measures are made as follows:

$$
\begin{gathered}
\theta(x, A)=f(x) \varphi(A) \\
\vartheta(x, A)=g(x) \omega(A)
\end{gathered}
$$


and functions $f, g: \mathbb{R}^{0} \rightarrow \mathbb{R}^{0}$ have (**) feature and $\varphi, \omega: 2^{X} \rightarrow \mathbb{R}^{0}$ were two measures. In addition, binary operations $(\sqcup, \Pi)$ is defines as follow:

$$
\begin{aligned}
& a \sqcup b=(a \otimes b)+(a \widehat{\otimes} b) \\
& a \sqcap b=(a \boxplus b)+(a \text { 刃 } b)
\end{aligned}
$$

Then $\mu: \mathbb{R}^{0} \times 2^{X} \rightarrow \mathbb{R}^{0}$ is defines as follow, It's a dependable value measure under the binary operation( $\left.\sqcup, \Pi\right)$.

$$
\mu(x, A)=(\theta(x, A))+(\vartheta(x, A))
$$

Proof.The consideration of function $\mu: \mathbb{R}^{0} \times 2^{X} \rightarrow \mathbb{R}^{0}$ is so simple. Now, we are going to prove the second feature. According to the hypothesis of the problem:

$$
\begin{aligned}
& \theta(x, A) \otimes \theta(y, B) \leq \theta(x \boxplus y, A \cup B) \\
& \vartheta(x, A) \widehat{\otimes} \vartheta(y, B) \leq \vartheta(x, \mathbb{\boxplus} y, A \cup B)
\end{aligned}
$$

As a result, we can write down the following conditions:

$$
[\theta(x, A) \otimes \theta(y, B)]+[\vartheta(x, A) \widehat{\otimes} \vartheta(y, B)] \leq[\theta(x \boxplus y, A \cup B)]+[\vartheta(x, \widehat{\boxplus} y, A \cup B)]
$$

According to the inequality and the features of (7) and (8) and (9) and (10) and due to the function $f, g$ has $(* *)$ feature, so we can write as follow:

$$
\begin{gathered}
\mu(x, A) \sqcup \mu(y, B)=[\theta(x, A) \otimes \theta(y, B)]+[\vartheta(x, A) \widehat{\otimes} \vartheta(y, B)] \\
\leq[\boldsymbol{\theta}(\boldsymbol{x} \boxplus \boldsymbol{y}, \boldsymbol{A} \cup \boldsymbol{B})]+[\boldsymbol{\vartheta}(\boldsymbol{x}, \widehat{\boxplus} \boldsymbol{y}, \boldsymbol{A} \cup \boldsymbol{B})]= \\
{[\boldsymbol{f}(\boldsymbol{x} \boxplus \boldsymbol{y}) \boldsymbol{\varphi}(\boldsymbol{A} \cup \boldsymbol{B})]+[\boldsymbol{g}(\boldsymbol{a} \boxplus \boldsymbol{\boxplus}) \boldsymbol{\omega}(\boldsymbol{A} \cup \boldsymbol{B})] \leq} \\
{[(\boldsymbol{f}(\boldsymbol{x} \boxplus \boldsymbol{y})+\boldsymbol{f}(\boldsymbol{x} \text { 开 } \boldsymbol{y})) \boldsymbol{\varphi}(\boldsymbol{A} \cup \boldsymbol{B})]+[(\boldsymbol{g}(\boldsymbol{a} \boxplus \boldsymbol{b})+\boldsymbol{g}(\boldsymbol{a} \widehat{\boxplus} \boldsymbol{b})) \boldsymbol{\omega}(\boldsymbol{A} \cup \boldsymbol{B})]} \\
\leq[\boldsymbol{f}((\boldsymbol{a} \boxplus \boldsymbol{b})+(\boldsymbol{a} \widehat{\boxplus} \boldsymbol{b})) \boldsymbol{\varphi}(\boldsymbol{A} \cup \boldsymbol{B})]+[\boldsymbol{g}((\boldsymbol{a} \boxplus \boldsymbol{b})+(\boldsymbol{a} \widehat{\boxplus} \boldsymbol{b})) \boldsymbol{\omega}(\boldsymbol{A} \cup \boldsymbol{B})]= \\
\boldsymbol{\theta}(\boldsymbol{x} \sqcap \boldsymbol{y}, \boldsymbol{A} \cup \boldsymbol{B})+\boldsymbol{\vartheta}(\boldsymbol{x} \sqcap \boldsymbol{y}, \boldsymbol{A} \cup \boldsymbol{B})=\boldsymbol{\mu}(\boldsymbol{x} \sqcap \boldsymbol{y}, \boldsymbol{A} \cup \boldsymbol{B})
\end{gathered}
$$

Notice.Let's think $\rho(x, A)=x^{2} m(A)$ is a dependable value measure . In this case, we can put $f(x)=\sqrt[86]{x}$ function instead of $x$. As a summary, the resulting function is no dependable value measure.

$$
\rho(\sqrt[86]{x}, A)=\sqrt[43]{x} \cdot m(A)
$$

That's why it can prove easily that the following relation is established according to the defined function.

$$
\rho(f(x), A)+\rho(f(y), A) \geq \rho(f(x+y, A))
$$

Therefore, as we can see in the example, by selecting a function we can change the direction of the inequality. In the following, we are looking for the conditions, which can choose the functions that don't change by placing them in dependable value measure of inequality.

Theorem 5.Let's imagine $\boldsymbol{\theta}: \mathbb{R}^{\mathbf{0}} \times \mathbf{2}^{\boldsymbol{X}} \rightarrow \mathbb{R}^{\mathbf{0}}$ is dependable value measure under binary operations $(\otimes, \boxplus)$. Also, if we have non-negative function $\boldsymbol{f}: \mathbb{R}^{0} \rightarrow \mathbb{R}^{0}$ and binary

operations $\Delta, \Pi$ such that:

$$
\begin{gathered}
a \sqcap b \leq \boldsymbol{a} \otimes \boldsymbol{b} \\
\boldsymbol{\theta}(\boldsymbol{f}(\boldsymbol{x}) \boxplus \boldsymbol{f}(\boldsymbol{y}), \boldsymbol{A}) \leq \boldsymbol{\theta}(\boldsymbol{f}(\boldsymbol{x} \Delta \boldsymbol{y}), \boldsymbol{A})
\end{gathered}
$$

Then function $\boldsymbol{\mu}: \mathbb{R}^{\mathbf{0}} \times \mathbf{2}^{X} \rightarrow \mathbb{R}^{\mathbf{0}}$ which define as follow is also dependable value measure under binary operations $(\Pi, \Delta)$.

Proof.It is sufficient to prove $\boldsymbol{I I}$ features.

$$
\mu(x, A)=\theta(f(x), A)
$$

$$
\begin{gathered}
\mu(x, A) \sqcap \mu(y, B) \leq \mu(x, A) \otimes \mu(y, B)=\theta(f(x), A) \otimes \theta(f(y), B) \leq \\
\theta(f(x) \boxplus f(y), A \cup B) \leq \theta(f(x \Delta y), A \cup B)=\mu(x \Delta y, A \cup B)
\end{gathered}
$$

Corollary. Let's think $\boldsymbol{\rho}: \mathbb{R}^{\mathbf{0}} \times \mathbf{2}^{X} \rightarrow \mathbb{R}^{\mathbf{0}}$ is dependable value measure. In addition, the negative function $\boldsymbol{\varphi}: \mathbb{R}^{\mathbf{0}} \times \mathbf{2}^{X} \rightarrow \mathbb{R}^{\mathbf{0}}$ satisfies in this $\left({ }^{* *}\right)$ condition. In this case, the function $\boldsymbol{\omega}: \mathbb{R}^{\mathbf{0}} \times \mathbf{2}^{X} \rightarrow \mathbb{R}^{\mathbf{0}}$ can define as follow, which is a dependable value measure.

$$
\omega(x, A)=\rho(\varphi(x), A)
$$


For example, from dependable value measure of $: \mathbb{R}^{\mathbf{0}} \times \mathbf{2}^{X} \rightarrow \mathbb{R}^{\mathbf{0}}$, we can make following dependable value measure :

$$
\rho(x, A)=\theta\left(\frac{x^{88}+61 x^{77}+4 x^{3}+5}{x+1}, A\right)
$$

We can easily recognize that function $\varphi(x)=\frac{x^{88}+61 x^{77}+4 x^{3}+5}{x+1}$ can satisfy $(* *)$ condition. Therefore, we can conclude that the function $\boldsymbol{\rho}: \mathbb{R}^{\mathbf{0}} \times \mathbf{2}^{X} \rightarrow \mathbb{R}^{\mathbf{0}}$ is dependable value measure.

Theorem6.suppose $\theta: \mathbb{R}^{0} \times 2^{X} \rightarrow \mathbb{R}^{0}$ be a dependable value measure under binary operations $(\Theta, \boxplus)$ and also functions $f, g: \mathbb{R}^{0} \rightarrow \mathbb{R}^{0}$ can satisfy the condition

$$
\boldsymbol{\theta}(\boldsymbol{f}(\boldsymbol{x}), \boldsymbol{A}) \geq \boldsymbol{\theta}(\boldsymbol{g}(\boldsymbol{x}), \boldsymbol{A})
$$

In addition, due to the binary operations $(\otimes, \boxplus)$, we have the following relations:

$$
\begin{aligned}
&(\boldsymbol{a}-\boldsymbol{b}) \otimes(\boldsymbol{c}-\boldsymbol{d}) \leq(\boldsymbol{a} \otimes \boldsymbol{c})-(\boldsymbol{b} \otimes \boldsymbol{d}) \\
&(\boldsymbol{\theta}(\boldsymbol{f}(\boldsymbol{x}), \boldsymbol{A}) \otimes(\boldsymbol{\theta}(\boldsymbol{f}(\boldsymbol{y}), \boldsymbol{B}))+(\boldsymbol{\theta}(\boldsymbol{g}(\boldsymbol{x} \boxplus \boldsymbol{y}), \boldsymbol{A} \cup \boldsymbol{B}) \\
& \leq(\boldsymbol{\theta}(\boldsymbol{f}(\boldsymbol{x} \boxplus \boldsymbol{y}), \boldsymbol{A} \cup \boldsymbol{B})+(\boldsymbol{\theta}(\boldsymbol{g}(\boldsymbol{x}), \boldsymbol{A}) \otimes \boldsymbol{\theta}(\boldsymbol{g}(\boldsymbol{y}), \boldsymbol{B}))
\end{aligned}
$$

In this case, function $\boldsymbol{\mu}: \mathbb{R}^{\mathbf{0}} \times \mathbf{2}^{X} \rightarrow \mathbb{R}^{\mathbf{0}}$ can define as follow which is a dependable value measure under binary operations $(\otimes, \boxplus)$.

Proof.According to feature (11), we conclude as follow:

$$
\mu(x, A)=\theta(f(x), A)-\theta(g(x), A)
$$

$$
\mu(\boldsymbol{x}, \boldsymbol{A})=\boldsymbol{\theta}(\boldsymbol{f}(\boldsymbol{x}), \boldsymbol{A})-\boldsymbol{\theta}(\boldsymbol{g}(\boldsymbol{x}), \boldsymbol{A}) \geq \mathbf{0}
$$

Now to continue, we prove the first feature.

$$
\begin{gathered}
\mu(x, A \cup B)=\theta(f(x), A \cup B)-\theta(g(x), A \cup B)= \\
\theta(f(x), A)+\theta(f(x), B)-\theta(g(x), A)-\theta(g(x), B)= \\
(\theta(f(x), A)-\theta(g(x), A))+(\theta(f(x), B)-\theta(g(x), B)= \\
\mu(x, A)+\mu(x, B)
\end{gathered}
$$

Now to prove the second feature, due to the inequalities (12) and (13), we have as follow:

$$
\begin{array}{r}
\mu(x, A) \otimes \mu(y, B)=(\theta(f(x), A)-\theta(g(x), A)) \otimes(\theta(f(y), B)-\theta(g(y), B)) \leq \\
(\theta(f(x), A) \otimes(\theta(f(y), B))-(\theta(g(x), A) \otimes \theta(g(y), B)) \leq \\
(\theta(f(x \boxplus y), A \cup B)-(\theta(g(x \boxplus y), A \cup B)=\mu(x \boxplus y, A \cup B)
\end{array}
$$

Notice.A sufficient condition for the inequality (13) is that functions $f, g: \mathbb{R}^{0} \rightarrow \mathbb{R}^{0}$ has the binary operations relations $(\otimes, \boxplus)$ :

$$
\begin{aligned}
& (\theta(f(x), A) \otimes(\theta(f(y), B)) \leq(\theta(f(x \boxplus y), A \cup B) \\
& (\theta(g(x), A) \otimes \theta(g(y), B)) \geq(\theta(g(x \boxplus y), A \cup B)
\end{aligned}
$$

Theorem7.Let's $\theta: \mathbb{R}^{0} \times 2^{X} \rightarrow \mathbb{R}^{0}$ is a dependable value measure under binary operations $(\otimes, \boxplus)$ and also functions $f, g: \mathbb{R}^{0} \rightarrow \mathbb{R}^{0}$ satisfy on (11) and (12) and (14) and (15) conditions. Then function $\mu: \mathbb{R}^{0} \times 2^{X} \rightarrow \mathbb{R}^{0}$ can define as a dependable value measure under binary operations $(\Theta, \boxplus)$.

$$
\boldsymbol{\mu}(\boldsymbol{x}, \boldsymbol{A})=\boldsymbol{\theta}(\boldsymbol{f}(\boldsymbol{x}), \boldsymbol{A})-\boldsymbol{\theta}(\boldsymbol{g}(\boldsymbol{x}), \boldsymbol{A})
$$

Example6.Let's think that $\theta: \mathbb{R}^{0} \times 2^{X} \rightarrow \mathbb{R}^{0}$ is a dependable value measure under binary operations $(\otimes, \boxplus)$ which define by following criterion.

$$
\theta(x, A)=x \cdot V(A)
$$

In addition, above binary operation should define as follow:

$$
\begin{gathered}
x \otimes y=y \\
x \boxplus y=x+y
\end{gathered}
$$

In this case, $\boldsymbol{\mu}: \mathbb{R}^{\mathbf{0}} \times \mathbf{2}^{X} \rightarrow \mathbb{R}^{\mathbf{0}}$ which define as criterion is a dependable value measure under binary operations $(\otimes, \boxplus)$. 


$$
\boldsymbol{\mu}(\boldsymbol{x}, \boldsymbol{A})=\boldsymbol{\theta}\left(\boldsymbol{x}+\boldsymbol{x}^{2}, \boldsymbol{A}\right)-\boldsymbol{\theta}(\boldsymbol{x}, \boldsymbol{A})
$$

Notice.In the previous theorem, both conditions are compulsory.

For example, you can consider the previous example $\boldsymbol{\mu}_{1}: \mathbb{R}^{\mathbf{0}} \times 2^{X} \rightarrow \mathbb{R}^{\mathbf{0}}$, which defined by below criterion:

$$
\mu_{1}(x, A)=\theta(x, A)-\theta\left(2 x+5 x^{2}, A\right)
$$

According to the defined criterion, it's clear that we have negative number for $\boldsymbol{x}$ which is a result of $\boldsymbol{\mu}_{\mathbf{1}}(\boldsymbol{x}, \boldsymbol{A})$.

Theorem8.Let's think $\theta_{i}: \mathbb{R}^{0} \times 2^{X} \rightarrow \mathbb{R}^{0}(1 \leq i \leq n)$ is a dependable value measures under binary operations $(\otimes, \boxplus)$ and these relations define as follow:

$$
\boldsymbol{\theta}_{i}(x, A)=f_{i}(x) V(A)
$$

In addition, we have this about binary operations $(\otimes, \boxplus)$ :

$$
\begin{gathered}
(a b) \otimes(c d) \leq(a \otimes c)(b \otimes d) \\
a \otimes b \leq \sqrt[n]{a^{n} \otimes b^{n}}
\end{gathered}
$$

In this case function $\boldsymbol{\mu}: \mathbb{R}^{\mathbf{0}} \times \mathbf{2}^{X} \rightarrow \mathbb{R}^{\mathbf{0}}$ can define as follow which is a dependable value measure under binary operations $(\otimes, \boxplus)$.

$$
\mu(x, A)=\prod_{i=1}^{n} \sqrt[n]{\theta_{i}(x, A)}
$$

Proof.The consideration of this feature(I), due to the above relations are too easy.

$$
\begin{gathered}
\mu(x, A \cup B)=\prod_{i=1}^{n} \sqrt[n]{\theta_{i}(x, A \cup B)}=\prod_{i=1}^{n} \sqrt[n]{f_{i}(x) V(A \cup B)}= \\
\sqrt[n]{\left(\prod_{i=1}^{n} f_{i}(x)\right)(V(A \cup B))^{n}=\prod_{i=1}^{n} \sqrt[n]{f_{i}(x)} V(A \cup B)=} \\
\sqrt[n]{\left(\prod_{i=1}^{n} f_{i}(x)\right)(V(A))^{n}+\sqrt[n]{f_{i}(x)}[V(A)+V(B)]=\prod_{i=1}^{n} \sqrt[n]{f_{i}(x)} V(A)+\prod_{i=1}^{n} \sqrt[n]{f_{i}(x)} V(B)=} \\
\sqrt{\left(\prod_{i=1}^{n} f_{i}(x)\right)(V(B))^{n}}=\prod_{i=1}^{n} \sqrt[n]{f_{i}(x) V(A)}+\prod_{i=1}^{n} \sqrt[n]{f_{i}(x) V(B)} \\
\prod_{i=1}^{n} \sqrt[n]{\theta_{i}(x, A)}+\prod_{i=1}^{n} \sqrt[n]{\theta_{i}(x, B)}= \\
\mu(x, A)+\mu(x, B)
\end{gathered}
$$

According to this feature (II) about (16) and (17), we can write as follow:

$$
\begin{gathered}
\mu(x, A) \otimes \mu(y, B)=\prod_{i=1}^{n} \sqrt[n]{\theta_{i}(x, A)} \otimes \prod_{i=1}^{n} \sqrt[n]{\theta_{i}(y, B)} \leq \\
\prod_{i=1}^{n}\left(\sqrt[n]{\theta_{i}(x, A)} \otimes \sqrt[n]{\theta_{i}(y, B)}\right) \leq \prod_{i=1}^{n}\left(\sqrt[n]{\theta_{i}(x, A) \otimes \theta_{i}(y, B)} \leq\right. \\
\prod_{i=1}^{n} \sqrt[n]{\theta_{i}(x \boxplus y, A \cup B)}=\mu(x \boxplus y, A \cup B)
\end{gathered}
$$

Lemma9.Let's think this function $\vartheta: \mathbb{R}^{0} \times 2^{X} \rightarrow \mathbb{R}^{0}$ is a dependable value pre-measure and also we have as follow:

$$
\begin{gathered}
\boldsymbol{\vartheta}(\boldsymbol{x}, \boldsymbol{A})+\boldsymbol{\vartheta}(\boldsymbol{y}, \boldsymbol{A}) \leq \boldsymbol{\vartheta}(\boldsymbol{x} \boxplus \boldsymbol{y}, \boldsymbol{A}) \\
\boldsymbol{a} \otimes \boldsymbol{b} \leq \boldsymbol{a}+\boldsymbol{b}
\end{gathered}
$$

In this case, $\boldsymbol{\vartheta}: \mathbb{R}^{\mathbf{0}} \times \mathbf{2}^{\boldsymbol{X}} \rightarrow \mathbb{R}^{\mathbf{0}}$ is a dependable value measure under binary operation $(\Theta, \boxplus)$.

Proof.Let's think this way:

$$
\begin{aligned}
& \boldsymbol{\vartheta}(\boldsymbol{x}, \boldsymbol{A})+\boldsymbol{\vartheta}(\boldsymbol{y}, \boldsymbol{A}) \leq \boldsymbol{\vartheta}(\boldsymbol{x} \boxplus y, A) \\
& \boldsymbol{\vartheta}(\boldsymbol{x}, \boldsymbol{B})+\boldsymbol{\vartheta}(y, B) \leq \boldsymbol{\vartheta}(\boldsymbol{x} \boxplus y, B)
\end{aligned}
$$

In order to addition of this relation, we can conclude as follow: 


$$
\boldsymbol{\vartheta}(\boldsymbol{x}, \boldsymbol{A})+\boldsymbol{\vartheta}(\boldsymbol{y}, \boldsymbol{B}) \leq \boldsymbol{\vartheta}(\boldsymbol{x} \boxplus \boldsymbol{y}, \boldsymbol{A} \cup \boldsymbol{B})
$$

Now, other operations are sole by (18) relation. Now, let's talk about a theorem which gives us an impressive test for examining the dependable value pre-measure.

Theorem10.Let's assume functions $\theta, \vartheta, \mu: \mathbb{R}^{0} \times 2^{X} \rightarrow \mathbb{R}^{0}$ and also suppose $\vartheta: \mathbb{R}^{0} \times 2^{X} \rightarrow \mathbb{R}^{0}$ is a dependable value pre-measure, Such that for each $x \in \mathbb{R}^{0}, A \subseteq X$ have relation:

$$
\boldsymbol{\theta}(\boldsymbol{x}, \boldsymbol{A}) \leq \boldsymbol{\vartheta}(\boldsymbol{x}, \boldsymbol{A}) \leq \boldsymbol{\mu}(\boldsymbol{x}, \boldsymbol{A})<\infty
$$

In addition, we have as follow:

$$
\boldsymbol{\mu}(\boldsymbol{x}, \boldsymbol{A}) \otimes \boldsymbol{\mu}(\boldsymbol{y}, \boldsymbol{A}) \leq \boldsymbol{\theta}(\boldsymbol{x} \boxplus \boldsymbol{y}, \boldsymbol{A})
$$

In addition, there should be binary operation $\otimes$ for (18) relations. Then $\boldsymbol{\vartheta}: \mathbb{R}^{+} \times \mathbf{2}^{X} \rightarrow \mathbb{R}^{+}$is a dependable value measure under binary operations $(\otimes, \boxplus)$.

Proof.It's clear that due to the assumptions and Lemma 10 to prove this relation $\vartheta(x, A) \otimes \vartheta(x, B) \leq$ $\vartheta(x \boxplus y, A \cup B)$, it's sufficient to prove that the following relation is established.

$$
\boldsymbol{\vartheta}(\boldsymbol{x}, \boldsymbol{A})+\boldsymbol{\vartheta}(\boldsymbol{x}, \boldsymbol{A}) \leq \boldsymbol{\vartheta}(\boldsymbol{x} \boxplus \boldsymbol{y}, \boldsymbol{A})
$$

In order to do this $\boldsymbol{p}(\boldsymbol{x}, \boldsymbol{A}), \boldsymbol{q}(\boldsymbol{x}, \boldsymbol{A})$ we have to define as follow;

$$
\begin{aligned}
& p(x, A)=\frac{\mu(x, A)+\theta(x, A)}{2} \\
& q(x, A)=\frac{\mu(x, A)-\theta(x, A)}{2}
\end{aligned}
$$

It's clear that we can write as follow:

$$
\begin{aligned}
& \theta(x, A)=p(x, A)-q(x, A) \\
& \mu(x, A)=p(x, A)+q(x, A)
\end{aligned}
$$

Therefore, we have as follow:

$$
p(x, A)-q(x, A) \leq \vartheta(x, A) \leq p(x, A)+q(x, A)
$$

So, we have as follow;

$$
\begin{gathered}
p(x \boxplus y, A)-q(x \boxplus y, A) \leq \vartheta(x \boxplus y, A) \\
-q(y, A)-p(y, A)-q(x, A)-p(x, A) \leq-\vartheta(y, A)-\vartheta(x, A)
\end{gathered}
$$

As a result, by addition of both unequal sides, we have as follow:

$$
\begin{gathered}
p(x \boxplus y, A)-(p(x, A)+p(y, A))-[q(x \boxplus y, A)+(q(x, A)+q(y, A))] \leq \\
\boldsymbol{\vartheta}(x \boxplus y, A)-[\vartheta(y, A)+\boldsymbol{\vartheta}(x, A)]
\end{gathered}
$$

Therefore, for prove $\boldsymbol{\vartheta}(\boldsymbol{x} \boxplus \boldsymbol{y}, \boldsymbol{A}) \geq[\boldsymbol{\vartheta}(\boldsymbol{y}, \boldsymbol{A})+\boldsymbol{\vartheta}(\boldsymbol{x}, \boldsymbol{A})]$ in above Inequality, there is sufficient condition to prove that:

$$
0 \leq p(x \boxplus y, A)-(p(x, A)+p(y, A))-[q(x \boxplus y, A)+(q(x, A)+q(y, A))]
$$

In order by replace the values, and streamline the relation, we can easily conclude as follow.

$$
\mu(x, A)+\mu(y, A) \leq \theta(x \boxplus y, A)
$$

For example, if $\boldsymbol{\tau}: \mathbb{R}^{\mathbf{0}} \times \mathbf{2}^{X} \rightarrow \mathbb{R}^{\mathbf{0}}$ is a dependable value measure. Such that relation $\boldsymbol{x} \geq \mathbf{1}$ is stable in order to $\boldsymbol{\mu}: \mathbb{R}^{\mathbf{0}} \times \mathbf{2}^{X} \rightarrow \mathbb{R}^{0}$. It can define as follow:

$$
\mu(x, A)=\frac{f(x) \cdot \tau\left(x^{2}-x, A\right)+g(x) \cdot \tau\left(x^{2}, A\right)}{f(x)+g(x)}
$$

Where $f, g: \mathbb{R}^{0} \rightarrow \mathbb{R}^{\mathbf{0}}$ and for every $x \in \mathbb{R}^{\mathbf{0}} f(x)+\boldsymbol{g}(\boldsymbol{x}) \neq \mathbf{0}$.

The proof that function $\boldsymbol{\mu}$ is dependable value pre-measure, it is easily.

According to the theorem 5, it's clear that $\boldsymbol{\tau}\left(\boldsymbol{x}^{2}-\boldsymbol{x}, \boldsymbol{A}\right)$ has this relation $\boldsymbol{x} \geq \mathbf{1}$ is a dependable value measure. In addition, according to the lemma 2 and criteria $\boldsymbol{\mu}: \mathbb{R}^{\mathbf{0}} \times \mathbf{2}^{X} \rightarrow \mathbb{R}^{\mathbf{0}}$, we have as follow;

$$
\tau\left(x^{2}-x, A\right) \leq \mu(x, A) \leq \tau\left(x^{2}, A\right)
$$

On the other hand, from $x, y \geq 1$, we can easily conclude that $2 x \boldsymbol{y} \geq \boldsymbol{x}+\boldsymbol{y}$. So, we have as follow; 


$$
\begin{gathered}
\tau\left(x^{2}, A\right)+\tau\left(y^{2}, A\right) \leq \tau\left(x^{2}, A\right)+\tau\left(y^{2}, A\right)+\tau(2 x y-x-y, A) \leq \tau\left(x^{2}+y^{2}+(2 x y-x-y), A\right) \\
=\tau\left((x+y)^{2}-(x+y), A\right)
\end{gathered}
$$

Therefore, due to the proven theorem, we conclude that $\boldsymbol{\mu}: \mathbb{R}^{\mathbf{0}} \times \mathbf{2}^{X} \rightarrow \mathbb{R}^{\mathbf{0}}$ is dependable value measure.

Theorem 11. Suppose that function $\mu: \mathbb{R}^{0} \times 2^{X} \rightarrow \mathbb{R}^{0}$ is a dependable value pre-measure and also we have this relation $x \leq y$ as follow:

$$
\mu(x, A) \leq \mu(y, A)
$$

In this case, $\boldsymbol{\mu}: \mathbb{R}^{\mathbf{0}} \times \mathbf{2}^{X} \rightarrow \mathbb{R}^{\mathbf{0}}$ is dependable value measure.

proof.

$$
\mu\left(x_{1}, A\right)+\mu\left(x_{2}, B\right) \leq \mu\left(x_{1}+x_{2}, A\right)+\mu\left(x_{1}+x_{2}, B\right)=\mu\left(x_{1}+x_{2}, A \cup B\right)
$$

Theorem 12.Let's think that the function $\boldsymbol{\vartheta}: \mathbb{R}^{\mathbf{0}} \times \mathbf{2}^{X} \rightarrow \mathbb{R}^{\mathbf{0}}$ is a dependable value pre-measure and $\boldsymbol{\mu}$ : $\mathbb{R}^{\mathbf{0}} \times \mathbf{2}^{\boldsymbol{X}} \rightarrow$ $\mathbb{R}^{0}$ is dependable value measure and we have this function $f: \mathbb{R}^{0} \times \mathbb{R}^{\mathbf{0}} \times \mathbf{2}^{X} \times \mathbf{2}^{X} \rightarrow \mathbb{R}^{\mathbf{0}}$ as follow:

In this case, $\boldsymbol{\vartheta}$ is a dependable value measure.

$$
\mu(y, B) \leq \vartheta(y, B) \leq \mu(x+y, A \bigcup B)-f(x, y, A, B)<\infty
$$

Proof. It's clear that we can as follow:

$$
\mu(y, B) \leq \vartheta(y, B) \leq \mu(x+y, A \bigcup B) \leq \vartheta(x+y, A \bigcup B)
$$

As a result, $\boldsymbol{\vartheta}: \mathbb{R}^{\mathbf{0}} \times \mathbf{2}^{X} \rightarrow \mathbb{R}^{\mathbf{0}}$ has One-sided feature and due to the previous theorem, we have a dependable value measure.

Example7.Let's think about the pre-measure $\vartheta: \mathbb{R}^{0} \times 2^{X} \rightarrow \mathbb{R}^{0}$ that can satisfy for any

arbitrary numbers $x, y \in \mathbb{R}^{0}$ and any sets $A, B \subseteq X$ of condition as follow:

$$
\boldsymbol{\vartheta}(\boldsymbol{y}, B) \leq \frac{\vartheta(x+y, A \cup B)}{f(x, y, A, B)}
$$

Such that $f: \mathbb{R}^{0} \times \mathbb{R}^{0} \times 2^{X} \times 2^{X} \rightarrow \mathbb{R}^{0}$ and

$$
\left\{\begin{array}{rr}
f(x, y, A, B) \geq 2 & \text { if } x \neq 0, A \neq \emptyset \\
f(x, y, A, B)=1 & \text { otherwise }
\end{array} \text { is turning to } \vartheta: \mathbb{R}^{0} \times 2^{X} \rightarrow \mathbb{R}^{0}\right. \text { which is dependable value measure. }
$$

Solution. Suppose that $x \neq 0, A \neq \emptyset$ hence we can use the condition of the problem, we can write as follow:

$$
\boldsymbol{\vartheta}(\boldsymbol{y}, \boldsymbol{B}) \leq \frac{\boldsymbol{\vartheta}(\boldsymbol{x}+\boldsymbol{y}, \boldsymbol{A} \cup \boldsymbol{B})}{2}
$$

Due to $\boldsymbol{x}, \boldsymbol{y}$, and desired numbers and sets $\boldsymbol{A}, \boldsymbol{B}$, we have as follow:

$$
\vartheta(x, A)+\vartheta(y, B) \leq \vartheta(x+y, A \bigcup B)
$$

In the following, we examine the feature of the dependable value measure, which can help us to accurately get the dependable value measure.

If $\boldsymbol{A}=\varnothing$ then we have as follow:

Theorem11 completes the answer.

$$
\boldsymbol{\vartheta}(\boldsymbol{y}, \boldsymbol{B}) \leq \boldsymbol{\vartheta}(\boldsymbol{x}+\boldsymbol{y}, \boldsymbol{B})
$$

Definition2.The dependable value measure of $\boldsymbol{\mu}: \mathbb{R}^{\mathbf{0}} \times \mathbf{2}^{X} \rightarrow \mathbb{R}^{\mathbf{0}}$, we call it has a simple form; If we have for every $\boldsymbol{x} \in \mathbb{R}^{\mathbf{0}}, \boldsymbol{A} \subseteq \boldsymbol{X}$ as follow:

$$
\boldsymbol{\mu}(\boldsymbol{x}, \boldsymbol{A})=\boldsymbol{f}(\boldsymbol{x}) \cdot \boldsymbol{V}(\boldsymbol{A})
$$

Such that $\boldsymbol{f}: \mathbb{R}^{\mathbf{0}} \rightarrow \mathbb{R}^{\mathbf{0}}$ can satisfy this condition (*), and also measure of $\boldsymbol{V}(\boldsymbol{A})$ is under $(\boldsymbol{X}, \boldsymbol{S}, \boldsymbol{V})$.

Theorem13.Assume that , $\mu: \mathbb{R}^{0} \times 2^{X} \rightarrow \mathbb{R}^{0}$ be a dependable value measure such that has a simple form. Also, suppose given deals $\mu(y, A), \mu(x, B)$ for constant number $x \in \mathbb{R}^{0}$, constant set $A \subseteq X$, any numbers $y \in \mathbb{R}^{0}$ and any sets such as $B \subseteq X$ and Also $\mu(x, \boldsymbol{A}) \neq \mathbf{0}$. Then for any arbitrary numbers such as $\boldsymbol{z} \in \mathbb{R}^{\mathbf{0}}$, and any arbitrary sets $\boldsymbol{C} \subseteq \boldsymbol{X} \boldsymbol{\mu}(\boldsymbol{z}, \boldsymbol{C})$ is calculable.

Proof.Let's think we have as follow:

$$
\mu(x, A)=f(x) \cdot V(A)
$$

In this case, we can conclude from this relation: 
Pay attention that $\boldsymbol{\mu}(\boldsymbol{x}, \boldsymbol{A}) \neq \mathbf{0}$ Therefore, we can calculate this $\boldsymbol{\mu}(\mathbf{z}, \boldsymbol{C})$ as follow:

$$
\begin{gathered}
\mu(z, C)=\frac{\mu(z, C) \times \mu(x, A)}{\mu(x, A)}=\frac{(f(z) \cdot V(C)) \times(f(x) \cdot V(A))}{\mu(x, A)}=\frac{(f(z) \cdot V(A)) \times(f(x) \cdot V(C)}{\mu(x, A)} \\
=\frac{\mu(z, A) \times \mu(x, C)}{\mu(x, A)}
\end{gathered}
$$

This simple theorem has important results. Some of them are referred to below.

Theorem 14. Suppose that we have below relation for some $B, C \subseteq X$ and

$$
\begin{gathered}
x, y, z \in \mathbb{R}^{0}: \\
\left\{\begin{array}{l}
\mu(y, C)>\mu(x, B)>0 \\
\mu(z, B)>\mu(y, D)>0
\end{array}\right.
\end{gathered}
$$

Then we have as follow:

$$
\boldsymbol{\mu}(\mathbf{z}, \boldsymbol{C})>\boldsymbol{\mu}(\boldsymbol{x}, \boldsymbol{D})>\mathbf{0}
$$

Proof.According to this $\mu(x, D), \mu(z, C) \neq 0$, we can use above theorem as follow:

$$
\begin{aligned}
& \mu(y, B)=\frac{\mu(y, C) \times \mu(z, B)}{\mu(z, C)} \\
& \mu(y, B)=\frac{\mu(y, D) \times \mu(x, B)}{\mu(x, D)}
\end{aligned}
$$

Therefore, we have as follow:

$$
\frac{\mu(y, C)}{\mu(x, B)} \times \frac{\mu(z, B)}{\mu(y, D)}=\frac{\mu(z, C)}{\mu(x, D)}
$$

This relation and hypothesis of theorem yield result.

Theorem 15.Let's think $\mu: \mathbb{R}^{0} \times 2^{X} \rightarrow \mathbb{R}^{0}$ is dependable value pre-measure. If for each numbers $a, b, x, y \in \mathbb{R}^{0}$ and for each sets like $A, B \subseteq X$ there exist $z, t \in \mathbb{R}^{0}$ and $C, D \subseteq X$, so we have as follow:

$$
\boldsymbol{a} \mu(z, A) \times \mu(x, C)+b \mu(y, D) \times \mu(t, B) \leq a b \mu(x+y, A \cup B)
$$

Also, the additional of $\boldsymbol{\mu}(\boldsymbol{z}, \boldsymbol{C}), \boldsymbol{\mu}(\boldsymbol{t}, \boldsymbol{D})$ be positive numbers. Then $\boldsymbol{\mu}: \mathbb{R}^{\mathbf{0}} \times \mathbf{2}^{\boldsymbol{X}} \rightarrow \mathbb{R}^{\mathbf{0}}$ is a dependable value measure.

Proof.According to the assumptions, we can write as follow:

$$
\frac{\mu(z, A) \times \mu(x, C)}{\mu(z, C)}+\frac{\mu(y, D) \times \mu(t, B)}{\mu(t, D)} \leq \mu(x+y, A \cup B)
$$

Now, due to the theorem 13, we can write as follow:

$$
\mu(x, A)+\mu(y, B) \leq \mu(x+y, A \cup B)
$$

Pay attention that assumptions are for $A, B \subseteq X$ and $x, y \in \mathbb{R}^{0}$, so this proof is over

Theorem 16.Let's $\mu: \mathbb{R}^{0} \times 2^{X} \rightarrow \mathbb{R}^{0}$ is dependable value pre-measure and also if for every numbers $a, b, x, y \in$ $\mathbb{R}^{0}$ and sets $A, B \subseteq X$, there exist a number $t \in \mathbb{R}^{0}$ and set $C \subseteq X$, such that :

$$
a \mu(x, A)+b \mu(y, B) \leq \mu(x+y, C) \times \mu(t, A \cup B)
$$

Then $\mu: \mathbb{R}^{0} \times 2^{X} \rightarrow \mathbb{R}^{0}$ is dependable value measure.

Proof. According to the assumptions, we can write as follow:

$$
a=b=\mu(t, C)
$$

Now, other operations by theorem 13 is simple.

\section{References}

Dean, R. A. (1996). Elements of Abstract Algebra. New York: Wily.

Finetti, D. B. (1994). Theory of probability (I),John wiley, New York.

Halmos, P. R., Measure, T., \& van Nostrand, (1997). NEW YORK,. 
Herstein, I. N. (1994). Topics in Algebra.New York :Blasdell.

Hewitt, E., Ross, K. A. (1965). Abstract Harmonic Analysis, I, Springer-Verlag, Berlin.

\section{Copyrights}

Copyright for this article is retained by the author(s), with first publication rights granted to the journal.

This is an open-access article distributed under the terms and conditions of the Creative Commons Attribution license (http://creativecommons.org/licenses/by/4.0/). 